\title{
miR-194-loaded gelatin nanospheres target MEF2C to suppress muscle atrophy in mechanical unloading model
}

Chen-Yan Zhang ${ }^{a, \#, *}$, Chang-Qing Yang, ${ }^{a, \#}$ Qiang Chen ${ }^{b, \#}$, Jie Liu $^{a}$, Ge Zhang ${ }^{a}$, Chen Dong ${ }^{a}$, Xin-Li Liu ${ }^{a}$, Hafiz Muhammad Umer Farooq ${ }^{a}$, Shi-Qi Zhao ${ }^{a}$, Li-Heng Luo , $^{2}$ Shan-Feng Jiang ${ }^{a}$, Yin-Bo Niu ${ }^{a}$, Da-Chuan Yin ${ }^{a} *$

a, Institute for Special Environmental Biophysics, Key Laboratory for Space Bioscience and Biotechnology, School of Life Sciences, Northwestern Polytechnical University, Xi'an 710072, Shaanxi, PR China

b, State Key Laboratory of Solidification Processing, Northwestern Polytechnical University, Xi'an 710072, Shaanxi, PR China

The authors declare no conflict of interest.

\footnotetext{
* To whom correspondence may be addressed. Chen-Yan Zhang email: zhangchenyan@nwpu.edu.cn, Tel: 86-29-88460543, Fax: 86-29-88460543; Da-Chuan Yin email: yindc@nwpu.edu.cn, Tel: 86-29-8860254, Fax: 86-29-8860254.
} 


\section{Supporting Information}

\begin{tabular}{|c|c|c|}
\hline \multicolumn{3}{|c|}{ SI Tables } \\
\hline SI 1 & Table S1. miR-194 mimic and inhibitor sequences & P3 \\
\hline SI 2 & Table S2. qPCR primer sequences in the experiment & P4- P5 \\
\hline \multicolumn{3}{|c|}{ SI Figures } \\
\hline SI 3 & $\begin{array}{l}\text { Figure } \mathbf{S 1} \text { The effect of miR-194 inhibitor on protein level of } \\
\text { myogenic markers and ubiquitin ligases in RPM C } 2 \mathrm{C} 12 \text { cells (a, } \\
\text { b) and muscle satellite cell }(\mathrm{c}, \mathrm{d}) \text { with differentiation medium. }\end{array}$ & P6 \\
\hline SI 4 & $\begin{array}{l}\text { Figure S2 Desmin immunofluorescence staining image of muscle } \\
\text { satellite cell. }\end{array}$ & $\mathrm{P} 7$ \\
\hline SI 5 & Figure $\mathbf{S 3}$ The mRNA expression of pax7 in muscle satellite cell. & P8 \\
\hline SI 6 & Figure S4 Particle size distribution and zeta potential of GNs. & P9 \\
\hline SI 7 & Figure S5 The size distribution of dry GNs nanosphere. & $\mathrm{P} 10$ \\
\hline SI 8 & $\begin{array}{l}\text { Figure S6 Myotube formation in the } \mathrm{C} 2 \mathrm{C} 12 \text { cells treated with the } \\
\text { GN. }\end{array}$ & P11 \\
\hline SI 9 & Figure S7 The effect of GNs on muscle development. & $\mathrm{P} 12$ \\
\hline
\end{tabular}


Table S1. miR-194 mimic and inhibitor sequences

\begin{tabular}{cc}
\hline Genes & Sequence \\
\hline Negative control & Forward: UUCUCCGAACGUGUCACGUTT \\
Reverse: & ACGUGACACGUUCGGAGAATT \\
miR-194 mimic & Forward: UGUAACAGCAACUCCAUGUGGA \\
miR-194 inhibitor & UCCACAUGGAGUUGCUGUUACA \\
\hline
\end{tabular}


Table S2. qPCR primer sequences in the experiment

\begin{tabular}{|c|c|}
\hline Genes & Sequences \\
\hline \multirow{3}{*}{ m-GAPDH } & Forward: TCGGTGTGAACGGATTTG \\
\hline & \\
\hline & Reverse: GGTCTCGCTCCTGGAAGA \\
\hline \multirow{3}{*}{ m-MEF2C } & Forward: ACCAGGACAAGGAATGGGAG \\
\hline & \\
\hline & Reverse: GGCGGCATGTTATGTAGGTG \\
\hline \multirow{3}{*}{ m-MyoD } & Forward: ACTTTCTGGAGCCCTCCTGGCA \\
\hline & \\
\hline & Reverse: TTTGTTGCACTACACAGCATG \\
\hline \multirow{3}{*}{ m-Myf5 } & Forward: ACTTTCTGGAGCCCTCCTGGCA \\
\hline & \\
\hline & Reverse: TTTGTTGCACTACACAGCATG \\
\hline \multirow[t]{2}{*}{ m-Myogenin } & Forward: CCCAACCCAGGAGATCATTT \\
\hline & Reverse: GTCTGGGAAGGCAACAGACA \\
\hline \multirow{3}{*}{ m-MyHC } & Forward: CAACTCCTTGATGCGTACGG \\
\hline & \\
\hline & Reverse: AGAATTGTCAGGAGCCACGA \\
\hline \multirow{3}{*}{ m-Atrogin-1 } & Forward: CTGAATAGCATCCAGATCAGCAGG \\
\hline & \\
\hline & Reverse: TTGATAAAGTCTTGAGGGGAAAGTG \\
\hline \multirow{2}{*}{ m-MuRF1 } & Forward: CAACTCCTTGATGCGTACGG \\
\hline & Reverse: AGAATTGTCAGGAGCCACGA \\
\hline \multirow{2}{*}{$\mathrm{m}-\mathrm{P} 50$} & Forward: TCGGAGACTGGAGCCTGTGGTG \\
\hline & Reverse: CCCTGCGTTGGATTTCGTGACT \\
\hline \multirow{3}{*}{$\mathrm{m}-\mathrm{Bcl} 3$} & Forward: CCTTTGATGCCCATTTACTCTA \\
\hline & \\
\hline & Reverse: AGCGGCTATGTTATTCTGGAC \\
\hline
\end{tabular}




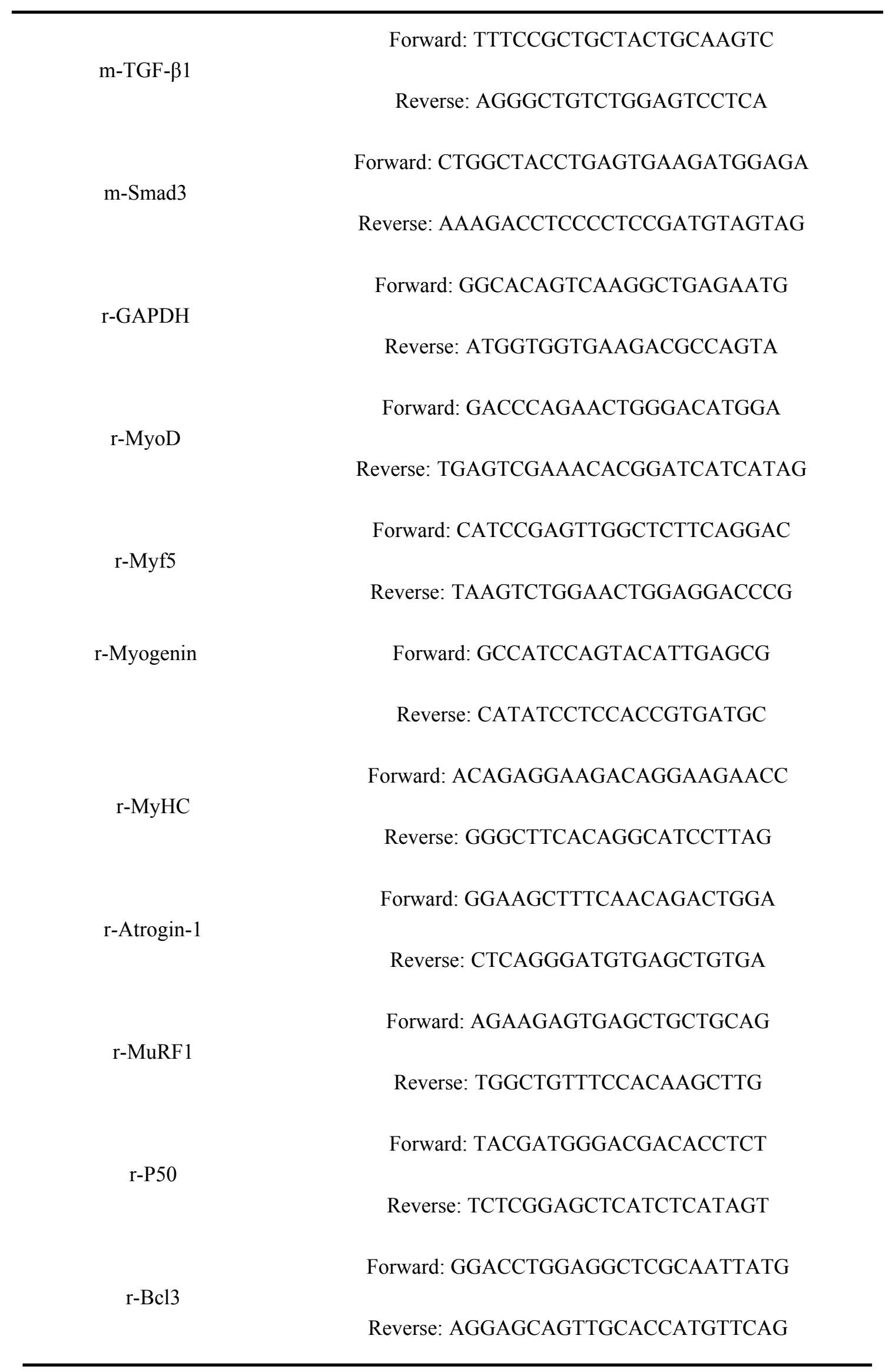


Forward: GGCACCATCCATGACATGAACCG

r-TGF- $\beta 1$

Reverse: GCCGTACACAGCAGTTCTTCTCTG

Forward: CTTCACAGCCGTCCATGACAGTAG

r-Smad3

Reverse: CCAATGTAGTAGAGCCGCACACC

Forward: GCGACGAATTCTGGAAAAAAAAAGT

m-MEF2C-WT-3'UTR

Reverse: GCGACTCTCGAGCGTGCTTGGATGT

Forward: AATGTATGCCACAATAGATAGTGTC

MEF2C-MUT-3'UTR

Reverse: TATCTATTGTGGCATACATTTTGCA 
$\mathbf{a}$ b

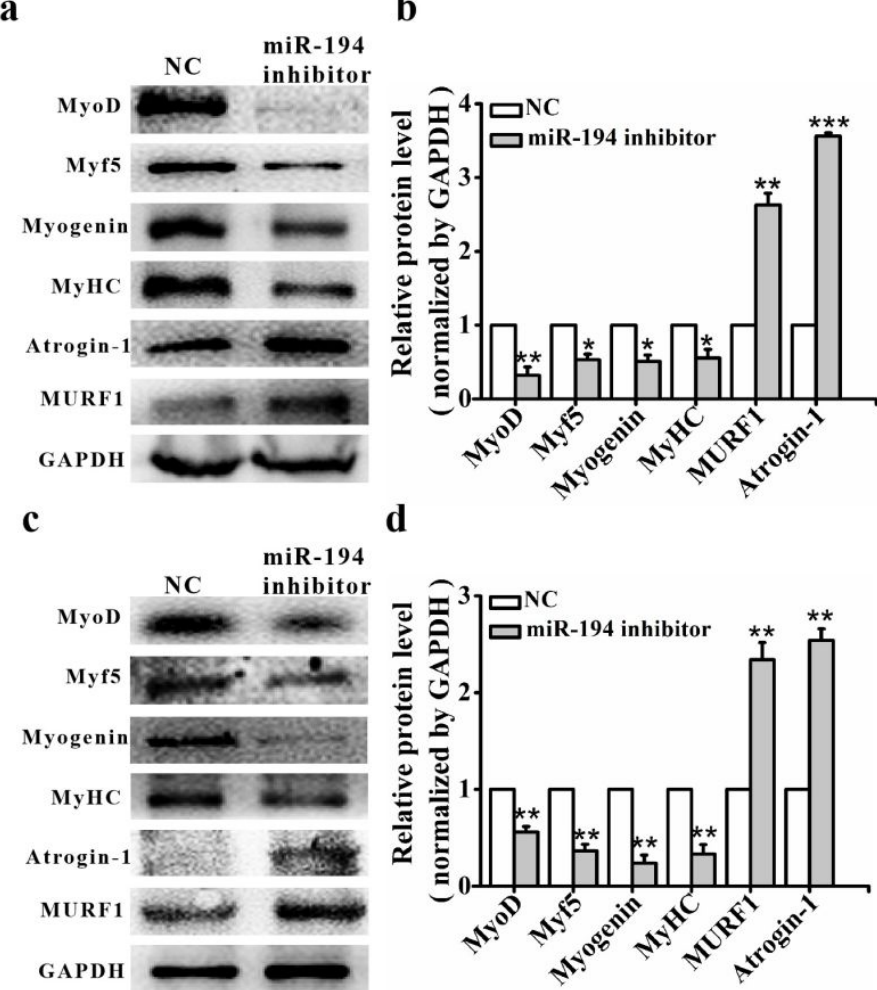

Figure S1 The effect of miR-194 inhibitor on protein level of myogenic markers and ubiquitin ligases in RPM C2C12 cells (a, b) and muscle satellite cell (c, d) after transfection for $48 \mathrm{~h}$. 
Figure S2 Desmin immunofluorescence staining image of muscle satellite cell. 


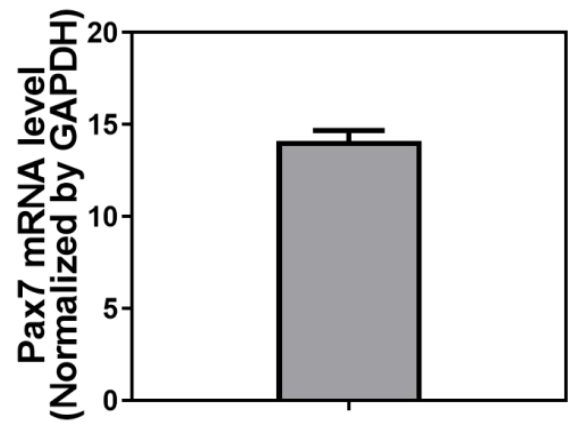

Figure S3 The mRNA expression of pax7 in muscle satellite cell. 


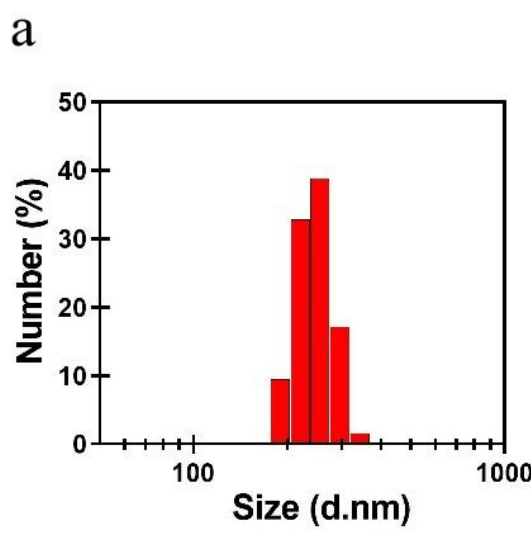

b

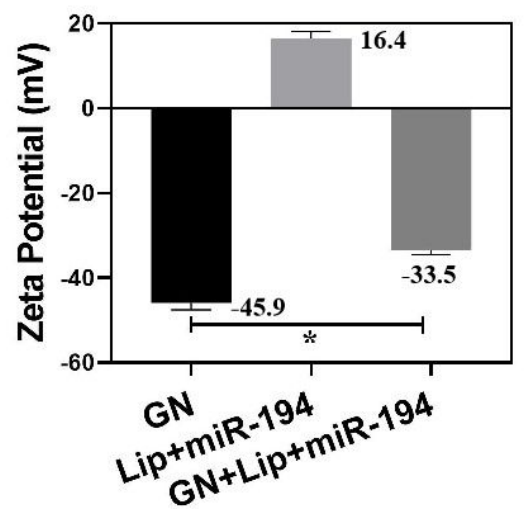

Figure S4 Particle size distribution and zeta potential of GNs. (a) Particle size distribution; (b) zeta potential. 


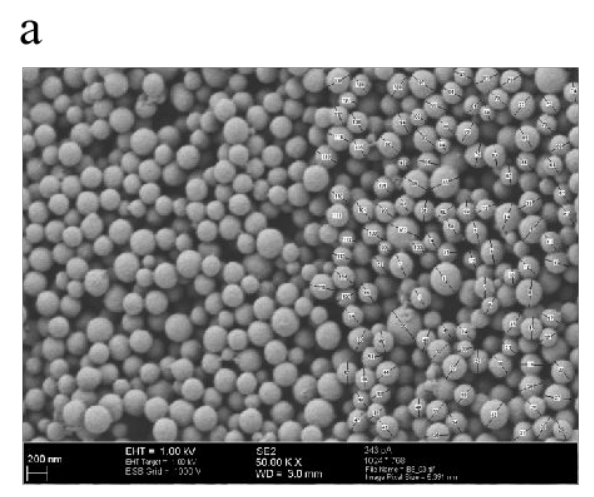

b

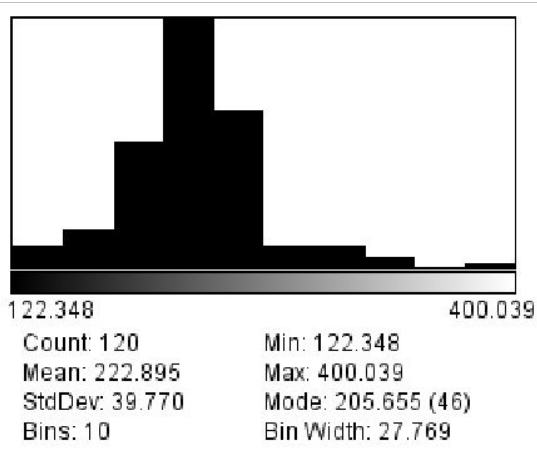

Figure S5 The size distribution of dry GNs nanosphere. (a) The size distribution of dry GNs nanospheres quantified from an SEM image; (b) The distribution of raw GNs. 

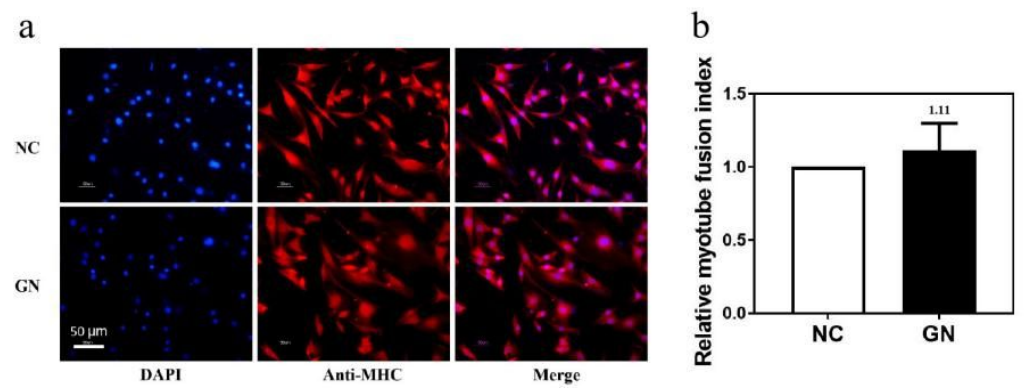

Figure S6 Myotube formation in the $\mathrm{C} 2 \mathrm{C} 12$ cells treated with the GN. 


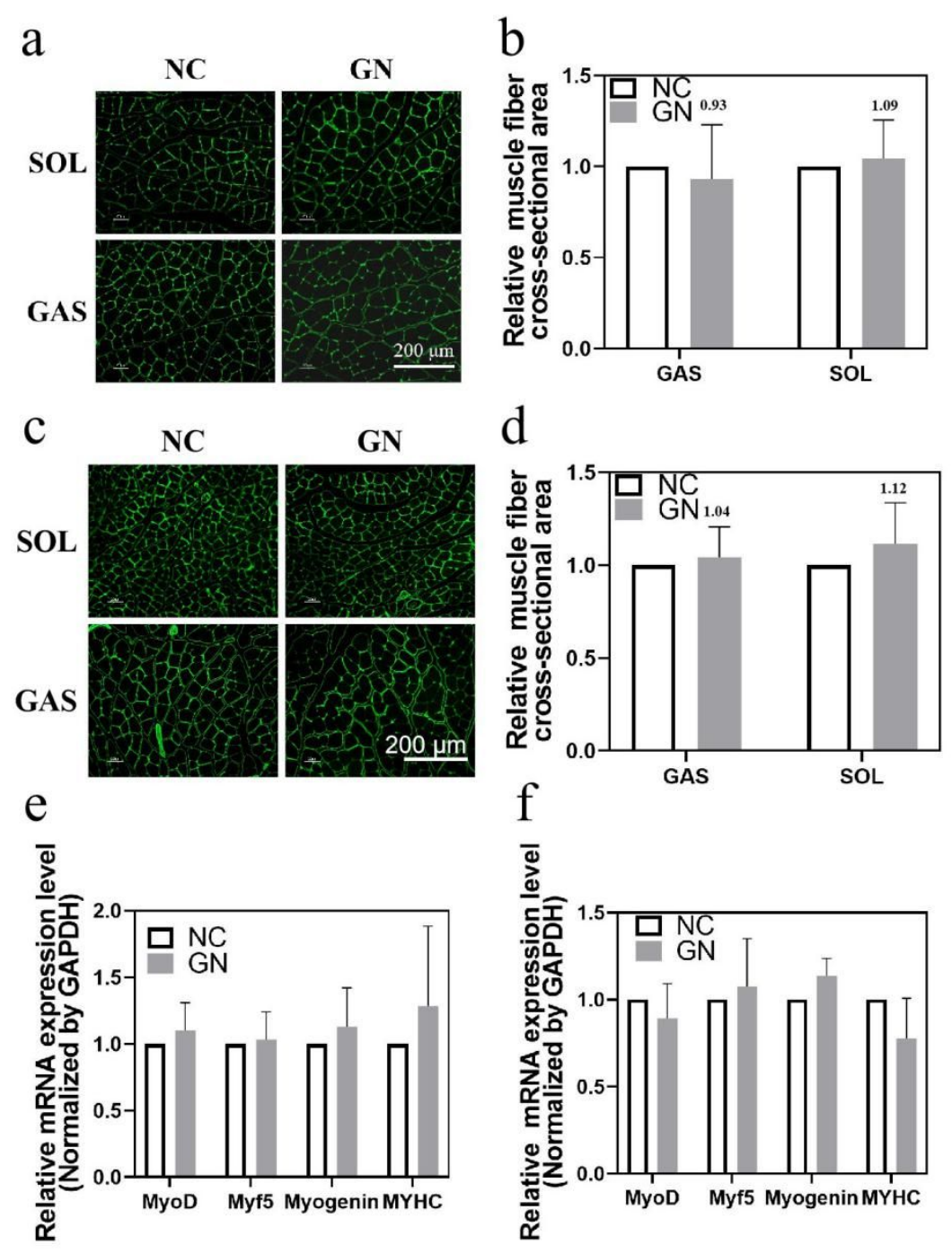

Figure S7 The effect of GNs on muscle development. (a) The effect of GNs on CAS of SOL (a) and GAS (b) in rats, SOL (c) and GAS (d) in mice, and expression of myogenetic markers in SOL (e) and GAS (f) of mice. 